\title{
Use of multiple low level features to find interesting regions
}

\author{
Michael Borck ${ }^{1,2}$, Geoff West ${ }^{1,2}$ and Tele Tan ${ }^{3,2}$ \\ ${ }^{1}$ Department of Spatial Sciences, Curtin University, Perth, Australia \\ ${ }^{2}$ CRC for Spatial Information, Australia \\ ${ }^{3}$ Department of Mechanical Engineering, Curtin University, Perth, Australia \\ michael.borck@postgrad.curtin.edu, \{g.west,t.tan\}@curtin.edu.au
}

Keywords: mobile mapping, feature selection, feature extraction, machine learning, 3D images

\begin{abstract}
Vehicle-based mobile mapping systems capture co-registered imagery and 3D point cloud information over hundreds of kilometres of transport corridor. Methods for extracting information from these large datasets are labour intensive and automatic methods are desired. In addition, such methods need to be easily configured by non-expert users to detect and measure many classes of objects. This paper describes a workflow to take a large number of image and depth features, use machine learning to generate an object detection system that is fast to configure and run. The output is high detection of the objects of interest but with an acceptable number of false alarms. This is desirable as the output is fed into a more complex and hence more computationally expensive analysis system to reject the false alarms and measure the remaining objects. Image and depth features from bounding boxes around objects of interest and random background are used for training with some popular learning algorithms. The interface allows a non-expert user to observe the performance and make modifications to improve the performance.
\end{abstract}

\section{INTRODUCTION}

Computer vision research is moving to the stage where quite complex systems can be used in real world applications, although in most cases the methods are turnkey. There are applications in which a non-expert user needs to configure a complex sequence of processes for their application. Such an application is the processing of co-registered imagery and $3 \mathrm{D}$ point cloud information acquired from a moving vehicle along transport corridors. GPS and inertial guidance allows the data to be registered to the world coordinate system enabling reasonably accurate location information to be acquired including the location of street side furniture, width of roads, power line to vegetation distances etc. Such systems can acquire enormous amounts of data quite quickly. For example the business district of Perth, Western Australia consists of $320 \mathrm{kms}$ of roads resulting in $122 \mathrm{GBytes}$ of image and depth data. Currently such data is processed manually meaning it can take months to analyse. The need is for methods to detect and measure objects of interest to the user from the co-registered imagery and depth data. Although 100\% detection with zero errors is desirable, however even if the performance is such that less human intervention is spent processing the data then that is a good outcome e.g. $100 \%$ detection and $20 \%$ false alarm rate.

This paper describes one stage of a multi-stage system to speed up the processing of mobile mapping data. A large number of image and depth features are extracted for objects of interest and the background. A number of classifiers are available that select the best combination of the features to give the best performance. The parameters of the system can be manipulated by the user to find all the objects of interest. The trade-off is a significant number of false alarms. However the overall result is that the number of regions that have to be further analysed is reduced meaning more complex and hence more computationally intensive methods can be used to increase performance. Results show that depth features improve the performance over just image features.

Although the main objective is good detection performance, the ease of use of such a system is also important, especially when integrated into the workflow of the user. Use is made of Orange, a GUI based open source interactive machine learning system (Demšar et al., 2004). Modules have been written to carry out image and depth processing and to interface to different data providing systems.

The main data capturing system used was Earth- 
mine, see Figure 1. The system captures panoramic imagery and uses stereo algorithms to generate coregistered 3D point clouds. Pairs of panoramic stereo images are captured typically at 10 metre spacing down a transport corridor. Each panorama is made up of images from four cameras pointing to the front, back, left and right of the vehicle. A 3D point cloud surrounds the position of the cameras uses a stereo template matching approach (Guinn, 2002).
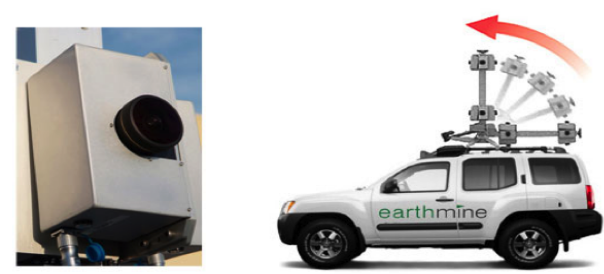

Figure 1: Earthmine system showing two sets of panorama cameras.

Earthmine has developed a server-based system that allows the querying via location to obtain data about a particular location. The data can be processed using a number of methods e.g. randomly, from a number of known locations, or by "driving" along the capture pathway.

The paper has two main contributions. First, there are questions on whether only image features or only depth features or some combination of image and depth should be used. Secondly the need to assess the performance of feature selection and feature extraction on a variety of classifiers. Results are presented that show the classification performance of the system on different objects and recommend appropriate features and classifiers.

\section{BACKGROUND}

It is very easy for a non-expert user to select regions from images using bounding boxes that isolate an object from the rest of the image. In most cases bounding boxes add in background information that can confuse recognition. Many features have been proposed that have a certain amount of "robustness" to the presence of background information as well as attempting to allow a certain amount of variation in the appearance of the object of interest. Features such as Haar wavelets and Histogram of Gradients (HoG) (Dalal and Triggs, 2005) have proven popular. Descriptors can be dense or use interesting points in the image. Some common interest points descriptors include Scale-Invariant Feature Transform (SIFT) (Lowe, 2004), Speed Up Ro- bust Features (SURF) (Bay et al., 2008) and Features from Accelerated Segment Test (FAST) (Rosten et al., 2010).

Similar to Alexe et al. (2010) it is assumed that an object possess a uniqueness that allows it to be segmented from the background and that this uniqueness can be learned. This research differs with this and other approaches as it includes the use of depth features. The question is which combination of these descriptors is best for each type of object that is required to be detected? In essence the more features to select from, the more likely the right combination will be found. Some feature are tuned to different circumstances e.g. it is widely regarded that HoG is very good for locating pedestrians but poor for other objects (Dalal and Triggs, 2005). Colour provides powerful information for object detection but the RGB channels are sensitive to lighting variations. In this paper intensity, hue and saturation features will be used. Statistical descriptors are quick to generate and provide a compact representation.

Texture is another salient feature in images ( $\mathrm{He}$ and Wang, 1991). Textures have been described by a precise statistical distribution of the image texture coming from the Gabor filter response (Wu et al., 2001), local binary patterns (Zhao and Pietikainen, 2006), and the Edge Histogram Descriptor (EHD) (Wu et al., 2001). Mikolajczyk and Schmid (2005) show that that moments and steerable filters, like Gabor filters, show the best performance among the low dimensional descriptors.

Interest points are often used to identify a point in an image that may be useful in image matching (Mikolajczyk and Schmid, 2001) and view-based object recognition (Lowe, 2004). This research focuses on trying to recognise generic classes. The proportion of interest points in a region is used as a descriptor under the premise that interesting regions will have a higher proportion of interest points.

Interesting regions will have more edges. It is not always possible to obtain ideal edges so encoding the proportion of edge pixels in a region is another measure to differentiate objects from non-objects (Phung and Bouzerdoum, 2007). Alexe et al. (2010) examine the edge density near the bounding box borders. In this research both of these features are implemented.

A range image contains distance measurements from a selected reference point or plane to surface points of objects within a scene (Besl, 1988) allowing more information of the scene to be recovered. Simply extending available descriptors designed for an intensity image to a depth image will not make use of additional information encoded in the depth map. Depth cues can improve object detection (Zhao et al., 
2012). It provides information about objects in terms of geometry and shape (Badami et al., 2013). Feature extraction on range images has proven to be more complex than on intensity images due to the irregular distribution of range image data and the nature of the features present in the range images (Coleman et al., 2007).

Surface normals and curvature estimates are extremely fast and easy to compute, they approximate the geometry of a point's k-neighborhood with only a few values. Similar to the orientation of edges, the orientation of surface normals provide additional information about the object. Histogram of Oriented Surface Normal (HoSN) (Tang et al., 2012) are designed to capture local geometric characteristics for object recognition with depth information. Local planarity compares the planes of neighbourhood regions by considering the relationship to the surrounding surface normals (Cadena and Košecka, 2013)

Zhao et al. (2012) propose a local depth pattern as a descriptor. They divide the region into cells, calculate the average depth of each cell and then calculate the difference between every cell pair. This difference vector forms the descriptor. Cadena and Košecka (2013) also use a depth difference feature that is calculated on superpixels rather than cells.

\section{WORKFLOW}

In many applications of computer vision, workflow is an important consideration. A user would typically read in some acquired data, process it interactively and produce the desired result. Much emphasis is on manual control of the process. Simple procedures can be fully automated if they are robust enough. However for many recognition applications, much tuning is needed by a skilled user. The challenge is to produce a workflow that a non-expert user can use to configure a complex process such as object detection. To do this, a user must be able to view selected parts of the data, identify objects of interest and train a system to use the best features for recognition through a feature selection process combined with some form of pattern recognition method such as a decision tree. Such a system was build using Orange, an open source toolkit for machine learning onto which image processing, visualisation and data acquisition methods were added. Figures 2 and 3 show an example of the workflow.

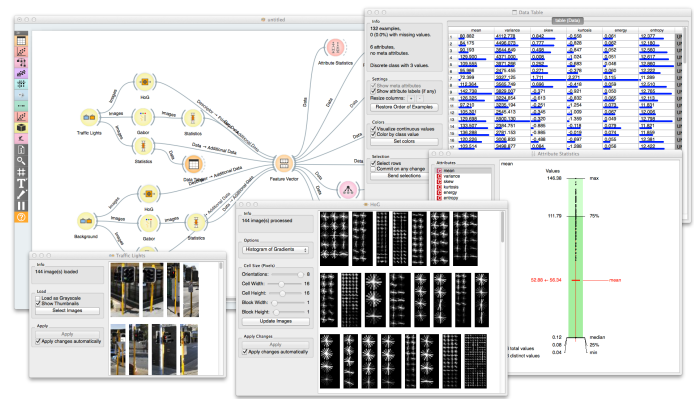

Figure 2: Example of image processing methods in Orange: Histogram of Gradients and statistical measures.

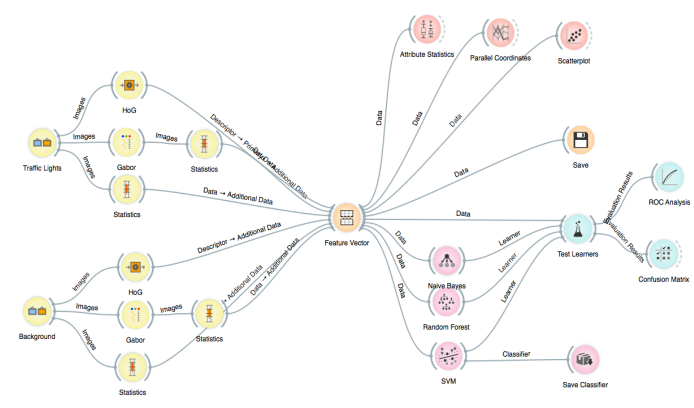

Figure 3: Full workflow in Orange from data through to classification performance.

\section{DATA}

Our data consists of a pairs of co-registered RGB and depth images. Within the Earthmine data set, 200 locations were visited. At each location each of the front, back, left and right tile views were obtained and bounding boxes defined to select objects of interest with the images. This resulted in a data set of 885 examples: 39 Bench seats; 53 Bus shelters; 69 Cars; 22 People; 81 Rubbish bins; 109 Street signs; 148 Traffic Lights; and 364 Background regions. Cars and people are considered dead space and would be removed.

Some of the background regions were selected to have similar aspect ratios to the objects as follows: 42 with aspect ratios similar to bus shelters; 93 with aspect ratios similar to rubbish bins; 117 with aspect rations similar to traffic lights; and 112 regions of random aspect ratios.

Figure 4 shows examples of intensity and depth data from the Earthmine system. For each of the four images: front, back, left and right, a depth image can be generated that is co-registered with the intensity image. In each of these images, darker pixels are further from the cameras. The intensity images are typical of street scenes and captured at shutter speeds that reduce blur as the vehicle could be travelling at 60-80 $\mathrm{kmph}$. The depth images can be seen to correspond to 
the intensity images especially with large scale parts of the environment. Small scale objects are picked out such as lamp posts. As with many stereo algorithms, features such as edges are relied upon to give the best estimation of depth from disparity and smooth areas require interpolation between reliable depth estimates to build a full depth map. The Earthmine data does produce confidence maps corresponding to the depth data although they are not currently used in this work.

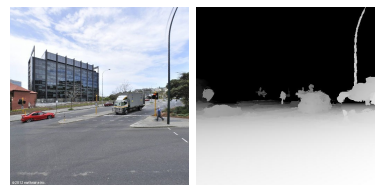

(a)

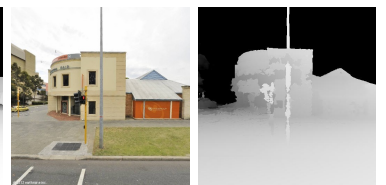

(b)
Figure 4: Example pairs of co-registered intensity and depth images (a) Image and depth map of an intersection. (b) Image and depth map of building.

Figures 5(a) to 5(h) show examples of coregistered intensity and depth images of the classes of objects used for object recognition.

In some images there is noise and a lack of good correspondence. Figure 5(a) shows a training image for traffic lights that have generally tall narrow bounding boxes. Figure 5(h) shows some of the training images for bus shelters that have generally a more broad aspect ratio. For all of these datasets, there is variation in appearance of each object.

Randomly acquired background regions enables the quick training of the system to discriminate between the objects of interest and the background.

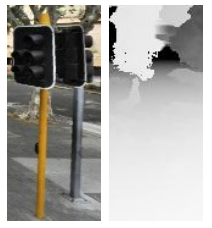

(a)

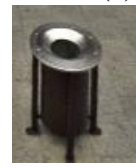

(e)

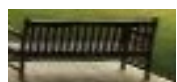

(g)

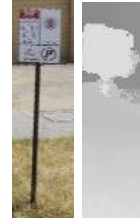

(b)

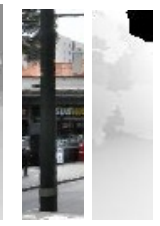

(c)

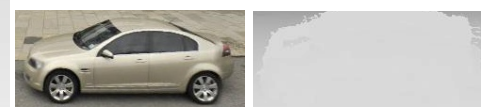

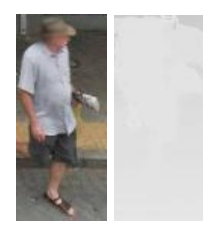

(d)

\section{(f)}

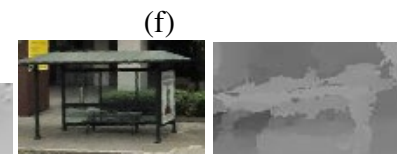

(h)

Figure 5: Example training image and corresponding depth map for eight classes. (a) Traffic light. (b) Street Sign. (c) Background. (d) Person. (e) Rubbish Bin. (f) Car. (g) Bench. (h) Bus Shelter.

\section{FEATURES}

For each bounding box a feature vector was constructed. For imagery intensity, hue and saturation channels were used. For each channel the following features are extracted: edge orientation histogram, mean, variance, skew, kurtosis, energy, entropy, edge density, Harris density, FAST density, local binary pattern encoded as a normalised histogram, and Gabor texture distribution encoded as mean and variance for angles 0, 45, 90 and 135 degrees.

The features above were also computed on the depth image when treated as a grey scale image. In addition depth specific features mean curvature, local planarity, an in-front-of feature, Histogram of Depth Difference (HoDD) and Histogram of Surface Normals (HoSN) were calculated. These are explained below.

Mean curvature was encoded as the mean and variance of the curvature across the region. Equation 4 from Kurita and Boulanger (1992) was used to calculate the curvature of a region.

Local planarity is computed using the dot product between the normal to the local plane against the kneighbourhood normals:

$$
1-\frac{1}{\|N\|} \sum n_{i} \cdot n_{j \in N}
$$

The in-front-of feature was encoded as the mean and standard deviation of the Local Depth Difference (LDD), where LDD is calculated between the depth at a pixel, $d_{i}$, and the k-neighbourhood's depths, $N$ :

$$
L D D= \begin{cases}\left\|d_{i}-d_{j \in N}\right\| & : d_{i}<\frac{1}{\|N\|} \sum\left(d_{j}\right)_{j \in N} \\ 0 & : \text { otherwise }\end{cases}
$$

To produce the HoDD, the region is divided into cells of fixed size. The difference of the average depth values of every cell pair is then calculated. This difference is recorded as a ten bin histogram. The histogram is then normalised to allow comparison of different size image regions.

For HoSN, each pixel in the region had a plane fitted to the k-neighbouring pixels. The angle of surface normal of the fitted plane with respect to the vertical plane was recorded in a histogram with bin size of $20^{\circ}$. The normalised histogram was used as the feature vector.

\section{EXPERIMENTAL PROCESS}

The objective is to find all objects of interest but with manageable false alarm rate. To learn which fea- 
tures and which classifier performs the best a classifier pipeline is built. The pipeline is used to explore the performance using a training set consisting of matching and non-matching image patches.

Classifiers considered include K-NN, Näive Bayes, Support Vector Machine (SVM), Decision Trees, Random Forests, Boosting, Bagging and Stacking. The last four are algorithms which use multiple models to improve prediction performance. Boosting and Bagging will be used to improve the weakest classifier identified during the preprocessing phase. For the stacking classifier all the classifiers except SVM were combined. The implementation and all details about these algorithms are available from the Orange data mining system.

Performance of machine learning methods may improve using a selected subset of best features. Feature selection is the process of selecting a subset of relevant features for use in model construction (Motoda and Liu, 2002). For this research a filter based on SVM weights was used and a feature subset selection wrapper based approach was written. The bespoke wrapper uses forward search, an induction algorithm, and search optimised for Area Under the Curve (AUC).

An alternative is to use Principal Components Analysis (PCA) to reduce the dimensionality of the feature vector. Principal Components (PCs) are selected by applying stopping rules (Jackson, 1993) thereby reducing the dimensionality of the data. A stopping rule is a decision criteria used to determine how many PCs to use. Four stopping rules used in this research include Kaiser-Guttman (Kaiser, 1960) and Scree Plot (Jackson, 1993), Broken-Stick and variance covered.

A discretisation algorithm is used to handle problems with real-valued attributes with Decision Trees and Bayesian Networks, treating the resulting intervals as nominal values. Learners use the MDLEntropy discretisation method provided in the Orange toolkit. For PCA and discretisation only the training data is used to determine the transform. The learned data transformation is applied to the test and validation data.

Half of the training data is used to tune classifiers prior to conducting any experiments. The automatic parameter search feature was used as provided by the Orange machine learning software. If no automatic feature was provided the default parameters were used. During this process a weak classifier from this initial process is identified to be used. On completion of tuning the parameters of each classifier remain fixed for all of the experiments.

It is reasonable to expect different combinations between feature representation and classifiers could yield different performance. Classifier Accuracy (CA) and AUC are two popular measures used to compare classifiers (Huang et al., 2003). Ling et al. (2003) and Yan et al. (2003) show the AUC is sufficient when comparing classifiers.

However, selecting models based on best AUC or CA can be misleading, especially if values are close. Repeating experiments can often end up with a slightly different values than previous runs. Methodologies are employed to reduce this effect, such as cross validation, but cannot eliminate the effect. Statistical tests are conducted to determine if differences in AUCs are statistically significant. For classifiers trained on the same data set the McNemar's test is used. McNemar's test cannot be used on classifiers trained on different data sets, so in this instance the nonparametric Wilcoxon signed rank test is used.

\section{RESULTS}

A number of scenarios were explored for various classifiers, classes and data sets. Data sets considered were image features, depth and a combination of image and depth features. Prior to training, principal components analysis and feature subset selection was applied to the data sets. A multiclass classification model was attempted to distinguish each on the classes in the one model. Classifiers built were based on K-NN, Näive Bayes, SVM, Decision Tree and Random Forest algorithm proved by the Orange toolkit. For each dataset a 10-fold cross-validation (using $70 \%$ of the data, 63:7) on each classifier was undertaken. Each classifier is tested for CA and AUC scores. Classifiers were ranked based on AUC and an approximate best model selected. Preliminary analysis rejected many classifiers. Tables 1 - 3 show the results for some of the better classifiers using different feature sets.

For the experiments, the best AUC for image features only was $0.967,0.919$ for depth features only, and 0.978 for combined image and depth features. The best classifier is the Support Vector Machine. Similar performance was observed from Random Forest classifiers (shown in bold in Tables 1-3). Within each feature set four classifiers with the highest AUCs were selected for McNemar testing. Table 4 clearly shows that SVM was the best classifier for the Filter(20), image + depth data set (see Table 3)

The best classifiers from each data set were ranked and a Wilcoxon signed rank test was performed pairwise on each set. For image features compared to image and depth the mean $p$-value was 0.317 with 
Table 1: Area Under Curve (AUC) and Classification Accuracy (CA) for image features. The number in brackets indicates how many features the classifier used.

\begin{tabular}{lcccccccc}
\hline & \multicolumn{2}{c}{ I+D (120) } & \multicolumn{2}{c}{ Filter (20) } & \multicolumn{2}{c}{ PCA (25) } & \multicolumn{2}{c}{ FSS-SVM (30) } \\
Classifier & AUC & CA & AUC & CA & AUC & CA & AUC & CA \\
\hline Bayes & 0.839 & 0.687 & 0.885 & 0.695 & 0.851 & 0.622 & 0.857 & 0.672 \\
Tree & 0.807 & 0.588 & 0.827 & 0.614 & 0.802 & 0.591 & 0.846 & 0.627 \\
kNN & 0.937 & 0.719 & 0.932 & 0.699 & 0.888 & 0.627 & 0.925 & 0.720 \\
SVM & $\mathbf{0 . 9 6 7}$ & $\mathbf{0 . 8 5 8}$ & $\mathbf{0 . 9 6 5}$ & $\mathbf{0 . 8 3 9}$ & $\mathbf{0 . 9 5 3}$ & $\mathbf{0 . 7 8 5}$ & $\mathbf{0 . 9 6 5}$ & $\mathbf{0 . 8 4 7}$ \\
Forest & $\mathbf{0 . 9 5 6}$ & $\mathbf{0 . 7 5 5}$ & $\mathbf{0 . 9 4 6}$ & $\mathbf{0 . 7 0 9}$ & $\mathbf{0 . 9 2 5}$ & $\mathbf{0 . 6 0 4}$ & $\mathbf{0 . 9 4 7}$ & $\mathbf{0 . 6 9 6}$ \\
Stacked & 0.949 & 0.776 & 0.935 & 0.724 & 0.914 & 0.661 & 0.940 & 0.727 \\
Boosted & 0.782 & 0.588 & 0.799 & 0.614 & 0.779 & 0.591 & 0.815 & 0.627 \\
Bagged & 0.910 & 0.722 & 0.907 & 0.698 & 0.890 & 0.677 & 0.910 & 0.733 \\
\hline
\end{tabular}

Table 2: Area Under Curve (AUC) and Classification Accuracy (CA) for depth features. The number in brackets indicates how many features the classifier used.

\begin{tabular}{lcccccccc}
\hline & \multicolumn{2}{c}{ I+D (66) } & \multicolumn{2}{c}{ Filter (20) } & \multicolumn{2}{c}{ PCA (13) } & \multicolumn{2}{c}{ FSS-Bayes (27) } \\
Classifier & AUC & CA & AUC & CA & AUC & CA & AUC & CA \\
\hline Bayes & 0.818 & 0.538 & 0.800 & 0.474 & 0.825 & 0.474 & 0.815 & 0.490 \\
Tree & 0.767 & 0.531 & 0.741 & 0.490 & 0.777 & 0.474 & 0.735 & 0.489 \\
kNN & 0.899 & 0.599 & 0.826 & 0.520 & 0.824 & 0.485 & 0.847 & 0.539 \\
SVM & $\mathbf{0 . 9 1 8}$ & $\mathbf{0 . 6 6 1}$ & $\mathbf{0 . 9 1 9}$ & $\mathbf{0 . 6 4 4}$ & $\mathbf{0 . 8 9 8}$ & $\mathbf{0 . 5 9 0}$ & $\mathbf{0 . 9 1 1}$ & $\mathbf{0 . 6 2 8}$ \\
Forest & $\mathbf{0 . 9 1 0}$ & $\mathbf{0 . 5 9 8}$ & $\mathbf{0 . 8 5 2}$ & $\mathbf{0 . 5 2 8}$ & $\mathbf{0 . 8 4 1}$ & $\mathbf{0 . 4 4 3}$ & $\mathbf{0 . 8 8 1}$ & $\mathbf{0 . 5 4 3}$ \\
Stacked & 0.896 & 0.596 & 0.837 & 0.505 & 0.813 & 0.474 & 0.857 & 0.510 \\
Boosted & 0.739 & 0.531 & 0.708 & 0.490 & 0.693 & 0.474 & 0.712 & 0.489 \\
Bagged & 0.869 & 0.611 & 0.812 & 0.559 & 0.799 & 0.516 & 0.826 & 0.585 \\
\hline
\end{tabular}

Table 3: Area Under Curve (AUC) and Classification Accuracy (CA) for image and depth features. The number in brackets indicates how many features the classifier used.

\begin{tabular}{lcccccccc}
\hline & \multicolumn{2}{c}{ I+D (186) } & \multicolumn{2}{c}{ Filter (20) } & \multicolumn{2}{c}{ PCA (25) } & \multicolumn{2}{c}{ FSS-kNN (34) } \\
Classifier & AUC & CA & AUC & CA & AUC & CA & AUC & CA \\
\hline Bayes & 0.823 & 0.678 & 0.886 & 0.687 & 0.876 & 0.656 & 0.877 & 0.695 \\
Tree & 0.815 & 0.615 & 0.841 & 0.617 & 0.796 & 0.546 & 0.839 & 0.646 \\
kNN & 0.962 & 0.767 & 0.936 & 0.711 & 0.898 & 0.643 & 0.953 & 0.769 \\
SVM & $\mathbf{0 . 9 7 8}$ & $\mathbf{0 . 8 5 9}$ & $\mathbf{0 . 9 7 5}$ & $\mathbf{0 . 8 4 3}$ & $\mathbf{0 . 9 7 1}$ & $\mathbf{0 . 8 1 3}$ & $\mathbf{0 . 9 7 8}$ & $\mathbf{0 . 8 5 5}$ \\
Forest & $\mathbf{0 . 9 6 9}$ & $\mathbf{0 . 7 7 3}$ & $\mathbf{0 . 9 5 2}$ & $\mathbf{0 . 7 0 6}$ & $\mathbf{0 . 9 3 6}$ & $\mathbf{0 . 6 0 6}$ & $\mathbf{0 . 9 6 0}$ & $\mathbf{0 . 7 4 3}$ \\
Stacked & 0.965 & 0.800 & 0.953 & 0.727 & 0.920 & 0.664 & 0.962 & 0.777 \\
Boosted & 0.811 & 0.615 & 0.812 & 0.617 & 0.767 & 0.546 & 0.812 & 0.646 \\
Bagged & 0.946 & 0.733 & 0.918 & 0.732 & 0.891 & 0.658 & 0.925 & 0.730 \\
\hline
\end{tabular}


Table 4: McNemar table of top four classifiers from the image and depth data set filtered for top twenty ranked features see column Filter(20) in Table 3.

\begin{tabular}{lrlc}
\hline & McNemar & $>3.84,5 \%$ & Best \\
\hline SVM vs Forest & 57.366 & Significant & SVM \\
SVM vs kNN & 50.469 & Significant & SVM \\
SVM vs Bagged & 37.593 & Significant & SVM \\
Forest vs kNN & 0.043 & Same & neither \\
Forest vs Bagged & 2.250 & Same & neither \\
kNN vs Bagged & 1.190 & Same & neither \\
\hline
\end{tabular}

a standard deviation of 0.048 . This indicates that there was no significant difference in discriminatory performance between image and the combination of depth and image. As there was no significant difference in AUCs, Filter (20), SVM, image + depth with the fewest number of features and better CA, was selected as the overall best classifier.

Using the best features, a Background vs Everything classifier was built to assess performance on detecting any class from the background. Figure 6 shows ROC curves for different classifiers and is consistent with previous results showing SVM, Random Forest and Näive Bayes being good classifiers.

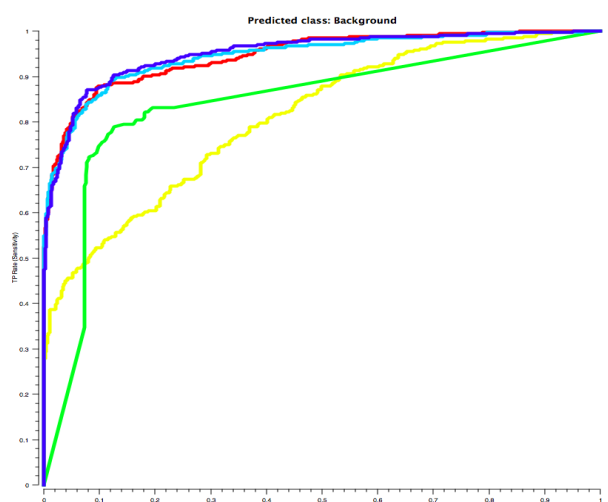

Figure 6: Background ROC curve for K-NN (Yellow), SVM (Purple), Decision Tree (Green), Random Forest (Aqua) and Näive Bayes (Red).

\section{CONCLUSIONS}

This paper has detailed an investigation into the first stage of a system for recognising objects present in co-registered image and depth data acquired from a vehicle based mobile mapping system. The depth data was acquired from the analysis of stereo pairs of panoramic images with errors present that are common in stereo analysis. The methods used reflect the need to find a technique that a non-expert user can use to train the system to do a relatively inexact recognition process to find all the objects of interest with a consequential significant but manageable false alarm rate. Bounding boxes are used to identify objects of interest as well as random background examples for training. A large number of features have been investigated with the thesis that machine learning will select the most useful ones. Features have been explored from traditional RGB images as well as from depth images using, in some cases, the same algorithms by regarding the depth images as monochrome grey scale images. The classification results show that image features perform better than depth features but a combination of image and depth features performs the best. The conclusion is that even quite coarse depth features can improve performance.

Future work will explore larger feature sets for each class, with more example classes. However it has to be noted that, for training, the smallest number of training examples is desired to improve the training workflow for the user.

\section{ACKNOWLEDGMENTS}

This work is supported by the Cooperative Research Centre for Spatial Information, whose activities are funded by the Australian Commonwealths Cooperative Research Centres Programme. It provides PhD scholarship for Michael Borck and partially funds Professor Geoff West's position. The authors would like to thank John Ristevski and Anthony Fassero from Earthmine and Landgate, WA for making available the datasets used in this work.

\section{REFERENCES}

Alexe, B., Deselaers, T., and Ferrari, V. (2010). What is an object? Computer Vision and Pattern Recognition, IEEE Computer Society Conference on.

Badami, I., Stückler, J., and Behnke, S. (2013). Depth-enhanced hough forests for object-class detection and continuous pose estimation. Semantic Perception, Mapping and Exploration, SPME2013.

Bay, H., Ess, A., Tuytelaars, T., and Gool, L. V. (2008). Speeded-up robust features (surf). Computer Vision and Image Understanding, 110(3):346 -359 .

Besl, P. J. (1988). Active, optical range imaging sensors. Machine vision and applications, 1(2):127152. 
Cadena, C. and Košecka, J. (2013). Semantic parsing for priming object detection in rgb-d scenes. In Semantic Perception, Mapping and Exploration (SPME) 2013.

Coleman, S., Scotney, B., and Suganthan, S. (2007). Feature extraction on range images - a new approach. In Robotics and Automation, 2007 IEEE International Conference on, pages 1098 -1103.

Dalal, N. and Triggs, B. (2005). Histograms of oriented gradients for human detection. In Schmid, C., Soatto, S., and Tomasi, C., editors, International Conference on Computer Vision \& Pattern Recognition, volume 2, pages 886-893.

Demšar, J., Zupan, B., Leban, G., and Curk, T. (2004). Orange: From experimental machine learning to interactive data mining. In Boulicaut, J.-F., Esposito, F., Giannotti, F., and Pedreschi, D., editors, Knowledge Discovery in Databases: PKDD 2004, pages 537-539. Springer.

Guinn, J. (2002). Enhanced formation flying validation report (jpl algorithm). NASA Goddard Space Flight Center Rept, pages 02-0548.

He, D.-C. and Wang, L. (1991). Texture features based on texture spectrum. Pattern Recognition, 24(5):391 - 399 .

Huang, J., Lu, J., and Ling, C. (2003). Comparing naive bayes, decision trees, and svm with auc and accuracy. In Data Mining, 2003. ICDM 2003. Third IEEE International Conference on, pages 553-556.

Jackson, D. A. (1993). Stopping rules in principal components analysis: a comparison of heuristical and statistical approaches. Ecology, pages 22042214.

Kaiser, H. F. (1960). The application of electronic computers to factor analysis. Educational and psychological measurement.

Kurita, T. and Boulanger, P. (1992). Computation of surface curvature from range images using geometrically intrinsic weights. MVA, pages 389-392.

Ling, C. X., Huang, J., and Zhang, H. (2003). Auc: a better measure than accuracy in comparing learning algorithms. In Advances in Artificial Intelligence, pages 329-341. Springer.

Lowe, D. G. (2004). Distinctive image features from scale-invariant keypoints. International Journal of Computer Vision, 60(2):91-110.
Mikolajczyk, K. and Schmid, C. (2001). Indexing based on scale invariant interest points. In Proceedings of Eighth IEEE International Conference on Computer Vision, 2001., volume 1, pages 525 -531 .

Mikolajczyk, K. and Schmid, C. (2005). A performance evaluation of local descriptors. IEEE Transactions on Pattern Analysis \& Machine Intelligence, 27(10):1615-1630.

Motoda, H. and Liu, H. (2002). Feature selection, extraction and construction. Communication of IICM (Institute of Information and Computing Machinery, Taiwan) Vol, 5:67-72.

Phung, S. and Bouzerdoum, A. (2007). Detecting people in images: An edge density approach. In Acoustics, Speech and Signal Processing, 2007. ICASSP 2007. IEEE International Conference on, volume 1, pages I-1229-I-1232.

Rosten, E., Porter, R., and Drummond, T. (2010). Faster and better: A machine learning approach to corner detection. Pattern Analysis and Machine Intelligence, IEEE Transactions on, 32(1):105 -119.

Tang, S., Wang, X., Lv, X., Han, T. X., Keller, J., He, Z., Skubic, M., and Lao, S. (2012). Histogram of oriented normal vectors for object recognition with a depth sensor. In Proceedings of 11th Asian Conference on Computer Vision (ACCV 2012).

Wu, P., Ro, Y., Won, C., and Choi, Y. (2001). Texture descriptors in mpeg-7. In Skarbek, W., editor, Computer Analysis of Images and Patterns, volume 2124 of Lecture Notes in Computer Science, pages 21-28. Springer Berlin Heidelberg.

Yan, L., Mozer, M. C., and Wolniewicz, R. (2003). Optimizing classifier performance via an approximation to the wilcoxon-mann-whitney statistic. Proceedings of the 20th International Conference on Machine Learning.

Zhao, G. and Pietikainen, M. (2006). Local binary pattern descriptors for dynamic texture recognition. In Pattern Recognition, 2006. ICPR 2006. 18th International Conference on, volume 2, pages 211214. IEEE.

Zhao, Y., Liu, Z., Yang, L., and Cheng, H. (2012). Combing rgb and depth map features for human activity recognition. In Signal Information Processing Association Annual Summit Conference (APSIPA ASC), 2012 Asia-Pacific, pages 1-4. 